\title{
406 - Spanish validation of the Revised Memory and Behavior Problems Checklist- Nursing Homes (RMBPC-NH)
}

Gema Perez-Rojo; Javier López; Cristina Velasco; Cristina Noriega; José Ángel Martínez-Huertas; Isabel Carretero; Patricia López Frutos; Leyre Galarraga

Introduction

The behavior problems in residents may affect professionals' performance at work, quality of work life, and even their health. Thus, it is important to have instruments that allow to estimate their prevalence. The objective of this study was to validate the Revised Memory and Behavior Problems ChecklistNursing Homes (RMBPC-NH; Allen et al., 2003) in a Spanish population. Specifically, it was tested the factor structure of the RMBPC-NH proposed by Wagner et al. (1995). Moreover, the relevance of the different types of problems for the working performance, at the level of individuals and institutions, was explored.

Method

In the present study, a total of 200 professionals participated.

Results

A Confirmatory Factor Analysis was conducted using WLSMV estimator in Mplus 7. Results showed a good fit to the data for the four-factor model $(\chi 2(813)=1733.73, \mathrm{p}<.001, \mathrm{CFI}=.90, \mathrm{TIL}=.90, \mathrm{RMSEA}=$ .08). Thus, it can be concluded that the original factor structure proposed by Wagner et al. (1995) and replicated by Allen et al. (2003) can also be applied to Spanish staff nursing homes. The reliability of the scale was adequate ( $\alpha$ from .86 to .93). Moreover, different descriptive and correlational results showed that both the factor scores of the Spanish adaptation of the RMBPC-NH and the importance of each type of problem were associated to different variable related.

Discussion

After analyzing the factor structure, reliability and validity of the adaptation of the RMBPC-NH scale for Spanish staff nursing homes it has found that it has good psychometric properties, so it could be a useful tool for this population.

This work was funded by the Spanish Ministry of Economy and Competitiveness (grant number PSI201679803-R).

Keywords: memory and behavior problems, old people, nursing homes, stress and job satisfaction 